\title{
Hipercolesterolemia familiar heterocigota: diagnóstico molecular y terapia hipolipemiante combinada. Caso clínico
}

\author{
Antonio Arteaga $\mathrm{LI}^{1}$, Ada Cuevas $\mathrm{M}^{2}$, Attilio Rigotti $\mathrm{R}^{3}$, \\ Francisco González ${ }^{4}$, Sergio Castillo ${ }^{5}$, Pedro Mata $L^{6}$, \\ Rodrigo Alonso $\mathrm{K}^{6}$. \\ Molecular diagnosis and combined \\ lipid lowering therapy of heterozygous \\ familial hypercholesterolemia. \\ Report of one case
}

\begin{abstract}
Heterozygous familial hypercholesterolemia affects one every 400 individuals, is caused by mutations in the LDL receptor gene and is associated with premature coronary artery disease. Nowadays, LDL cholesterol can be efficiently reduced with the new therapies to reduce blood lipids. We report a female patient who consulted in 1975, when she was 46 years old, for severe hypercholesterolemia. In 2003, a sample of leukocyte DNA was obtained and the uncommon $1705+1 \mathrm{G}>\mathrm{A}$ mutation of the $\mathrm{LDL}$ receptor gene was detected. No mutations in the apolipoprotein B gene were found. The patient was treated successfully with simvastatin $80 \mathrm{mg} /$ day and ezetimibe $10 \mathrm{mg} /$ day and LDL cholesterol levels were reduced below 200 mg/dl (Rev Méd Chile 2007; 135: 216-20).

(Key-words: Ezetimibe; Hypercholesterolemia, familial combined; LDL receptor related proteins; Simvastatin)

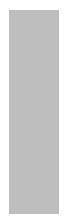

Recibido el 17 de enero, 2006. Aceptado el 22 de junio, 2006.

${ }_{1}^{1}$ Departamentos de Diabetes, Nutrición y Metabolismo y ${ }^{3}$ Gastroenterología, Facultad de Medicina, Pontificia Universidad Católica de Chile. ${ }^{2}$ Departamento de Medicina, Clínica Las Condes; Santiago, Chile. ${ }^{4}$ Laboratorio de Biología Molecular, Facultad de Medicina, Universidad de Valparaíso, Valparaíso, Chile. ${ }^{5}$ Laboratorio de Diagnóstico Genético Lácer, Barcelona, España. ${ }^{6}$ Clínica de Lípidos, Fundación Jiménez Díaz. Madrid, España.
\end{abstract}

L a hipercolesterolemia familiar (HF) es una enfermedad genética autosómica dominante causada por mutaciones en el gen que codifica el receptor de LDL (rLDL) localizado en el cromoso-

Correspondencia a: Dr. Antonio Arteaga Ll. Departamento de Diabetes, Nutrición y Metabolismo, Facultad de Medicina, Pontificia Universidad Católica. Marcoleta 367, Santiago, Chile. Fono: 56-2-6863861. E mail: aarteaga@med.puc.cl ma $19^{1}$. La consecuencia de este trastorno es una reducción importante en el número de receptores funcionales para las LDL a nivel hepático, por lo que se produce un aumento en las concentraciones plasmáticas de colesterol transportado en las lipoproteínas de baja densidad (c-LDL), asociado al depósito de colesterol en los tendones y al desarrollo de enfermedad cardiovascular prematura, especialmente cardiopatía isquémica ${ }^{2}$. 
La HF ha sido descrita en todo el mundo, y afecta a uno de cada 400-500 sujetos en su forma heterocigota y a 1 por cada 1.000 .000 de habitantes en su forma homocigota ${ }^{3}$. En nuestro país, nuestro grupo describió un caso clínico de HF en 1979, aunque no se realizó un estudio genético específico y el paciente no pudo ser tratado adecuadamente porque se carecía de tratamiento hipolipemiante efectivo ${ }^{4}$.

En esta comunicación, describimos el primer caso de HF con diagnóstico genético en Chile, asociado con una buena respuesta al tratamiento hipolipemiante combinado de simvastatina y ezetimiba.

\section{CASO CĹNICO}

CW, paciente de origen polaco y residente en Chile, consultó por primera vez en 1975 a la edad de 46 años por un cuadro de hipercolesterolemia fenotipo IIa, con concentraciones séricas de colesterol total de $524 \mathrm{mg} / \mathrm{dl}$ y triglicéridos de $116 \mathrm{mg} /$ dl. La paciente no refería sintomatología atribuible a patología cardiovascular isquémica coronaria, cerebral o periférica.

En sus antecedentes familiares destacaba que su madre y abuela materna padecieron de hipercolesterolemia aislada y cardiopatía coronaria precoz (manifestación clínica antes de los 65 años). En sus antecedentes personales, la paciente refería historia de TBC pulmonar, enfermedad reumática en su juventud y menopausia reciente. No fumaba ni bebía alcohol, tenía una vida activa, aunque sin actividad física programada y presentaba un consumo elevado de grasas saturadas.

El examen físico realizado en la consulta inicial mostró un índice de masa corporal de $27,8 \mathrm{~kg} / \mathrm{m}^{2}$ y presión arterial de 130/80 $\mathrm{mm}$ de $\mathrm{Hg}$. El examen cardíaco fue normal. No existían soplos carotídeos ni abdominal y los pulsos arteriales periféricos de las extremidades inferiores eran normales. La paciente tenía arco corneal bilateral y xantomas en los tendones extensores de las manos y en los tendones de Aquiles. El examen de fondo de ojo reflejaba una esclerosis leve de los vasos retineanos.

En los exámenes de laboratorio complementarios, la glicemia de ayuno fue de $85 \mathrm{mg} / \mathrm{dl}$ y la TSH de 2,8 uUI/ml con pruebas hepáticas y renales normales. Un electrocardiograma de esfuerzo resultó negativo para insuficiencia coronaria. El ultrasonido Doppler reveló ateromatosis no estenosante de ambas arterias carótidas internas y de las arterias subclavias.

Inicialmente, se indicó un régimen hipocalórico con reducción del consumo de grasas saturadas y colesterol e incremento de la ingesta de grasas poliinsaturadas y fibra dietética, sin obtenerse cambios significativos en las concentraciones de colesterol plasmático total (rango entre 430 y $500 \mathrm{mg} / \mathrm{dl}$ ) durante un año de seguimiento (Figura 1A). Posteriormente, se inició terapia con colesteramina comenzando con $4 \mathrm{~g} /$ día titulando la dosis hasta $24 \mathrm{~g} /$ día. La respuesta a esta resina fue dosis dependiente, aunque con un efecto hipocolesterolemiante muy discreto, alcanzando concentraciones de colesterol total sérico entre 300-400 mg/dl. Con las dosis más altas, la paciente refirió intolerancia digestiva, lo que obligó a reducir periódicamente la dosis de la colestiramina. Para reforzar el efecto de la resina, se asociaron fibratos (clofibrato, bezafibrato), sin obtenerse un efecto hipolipemiente adicional. En este momento, las concentraciones estimadas de c-LDL sérico oscilaban entre 300 y $350 \mathrm{mg} / \mathrm{dl}$ (Figura 1B).

En 1992, y con la introducción de las estatinas al arsenal terapéutico, la paciente comenzó monoterapia con lovastatina $40 \mathrm{mg} /$ día, sin obtener una respuesta significativa, aunque la asociación de colesteramina (8 g/día), permitió por primera vez reducir el c-LDL sérico a concentraciones $<300$ $\mathrm{mg} / \mathrm{dl}$ (Figura 1B). Desde entonces, se mantuvo con terapia hipolipemiante asociada (atorvastatina $20 \mathrm{mg} /$ día o simvastativa $80 \mathrm{mg} /$ día más colesteramina $8 \mathrm{~g} /$ día), alcanzándose con esta última combinación concentraciones de c-LDL entre 200$250 \mathrm{mg} / \mathrm{dl}$. Las concentraciones de triglicéridos y de colesterol HDL han oscilado dentro de rangos aceptables, excepto cuando la paciente presentó sobrepeso. Las transaminasas y la creatinfosfokinasa (CPK) total y fracción muscular plasmáticas han sido siempre normales.

Durante su evolución, la paciente desarrolló hipertensión arterial leve controlada con amlodipino (10 mg/día). Además, ha presentado episodios vertiginosos, comprobándose daño del VIII par craneano, posiblemente de naturaleza isquémica. Un control ecográfico carotídeo realizado el año 


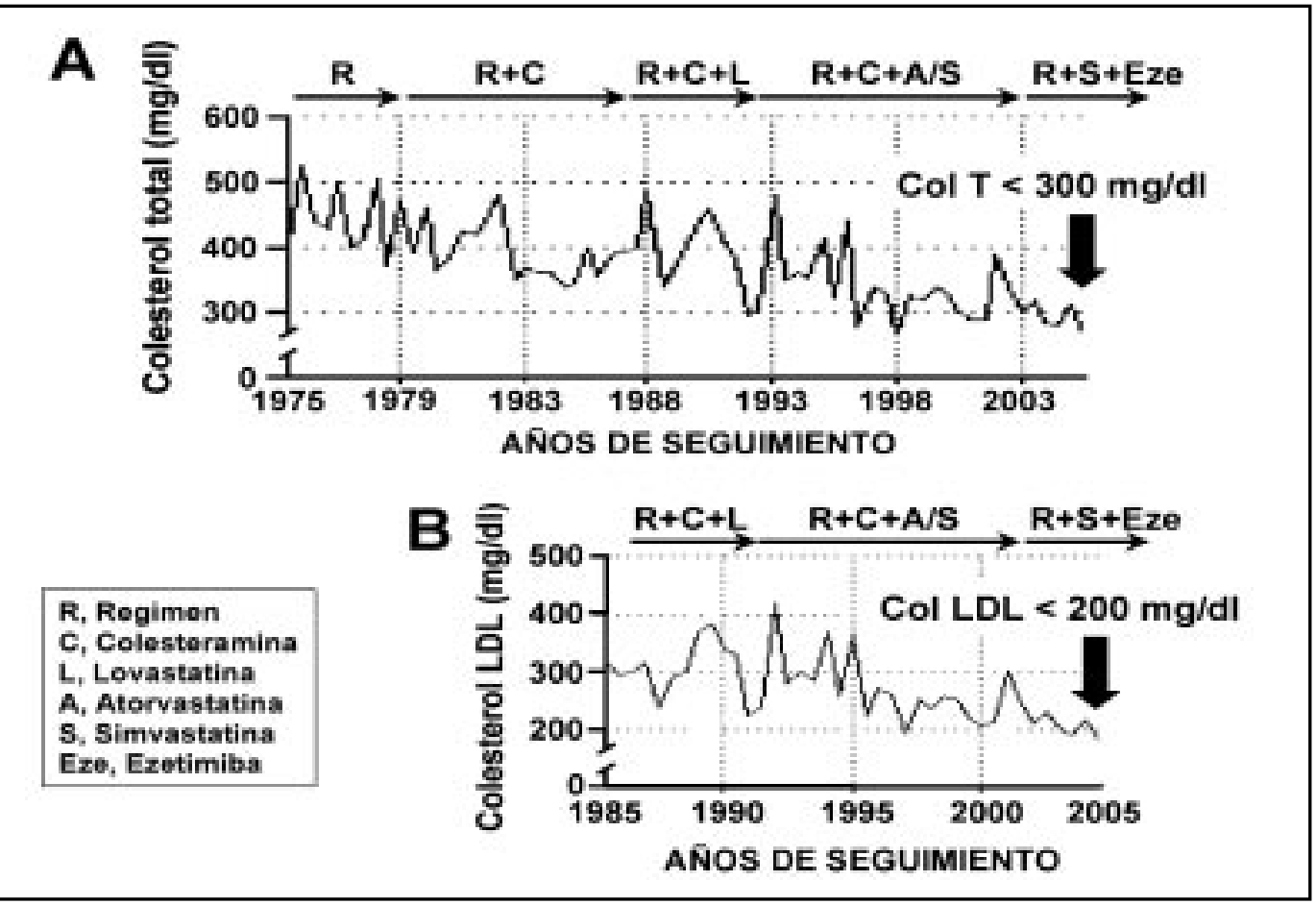

Figura 1. Efecto de diferentes terapias hipolipemiantes en un caso clínico de hipercolesterolemia familiar heterocigota en control desde 1975 a 2005. El panel A muestra la evolución de los niveles de colesterol sérico total, mientras que el panel B corresponde a los valores de colesterol LDL medidos en la paciente durante la aplicación de diferentes alternativas terapéuticas hipocolesterolemiantes.

2000 mostró ateromatosis carotídea bilateral con leve elongación de los vasos sin compromiso hemodinámico significativo. Un nuevo electrocardiograma de esfuerzo en 2002 fue negativo para insuficiencia del riego coronario. En ese mismo año, la paciente presentó tromboembolismo pulmonar asociado a viajes prolongados por lo cual inició terapia anticoagulante oral. En esa época y mientras la paciente estaba en tratamiento con simvastatina $80 \mathrm{mg} /$ día y con niveles de c-LDL de $242 \mathrm{mg} / \mathrm{dl}$, se obtuvo una muestra de ADN leucocitario para análisis molecular del gen del rLDL5, detectándose la mutación 1705 +1G >A, sin alteraciones en la secuencia del gen de la apolipoproteína B.

Desde hace un año, la paciente se encuentra en tratamiento con simvastatina $80 \mathrm{mg} /$ día + ezetimibe $10 \mathrm{mg} /$ día, alcanzando por primera vez concentraciones de LDL-C $\leq 200 \mathrm{mg} / \mathrm{dL}$ de forma persistente
(Figura 1B). En su último control en junio de 2005, la paciente se encontraba activa, sin sintomatología cardiovascular asociada y con buena capacidad física, presión arterial de 150/60 mmHg y el resto del examen físico sin variaciones significativas. La paciente está recibiendo diariamente simvastatina/ ezetimiba (80 mg/10 mg), amlodipino (10 mg) y acenocumarol. El perfil lipídico más reciente muestra un colesterol total de $260 \mathrm{mg} / \mathrm{dl}$, c-LDL de 180 $\mathrm{mg} / \mathrm{dl}$, colesterol HDL de $60 \mathrm{mg} / \mathrm{dl}$ y triglicéridos de $100 \mathrm{mg} / \mathrm{dl}$. El laboratorio adicional evidenció concentraciones plasmáticas normales de glucosa, transaminasas y CPK.

\section{DisCUSIÓN}

Presentamos el primer caso de HF diagnosticado mediante estudio genético en Chile y como el 
tratamiento hipolipemiante combinado con simvastatina y ezetimiba ha permitido que la paciente alcance por primera vez concentraciones de c-LDL inferiores a $200 \mathrm{mg} / \mathrm{dL}$, después de 30 años de evolución desde el diagnóstico clínico de su enfermedad.

El método utilizado para el diagnóstico genético fue el micro-array de ADN desarrollado en España, que tiene una sensibilidad y especificidad cercanas a $100 \%{ }^{5}$. Desde un punto de vista molecular, esta paciente presenta una mutación puntual en condición de heterocigosis en el gen del rLDL. La mutación se denomina $1705+1 \mathrm{G}>\mathrm{A}$, y fue descrita por Jensen et al en población danesa en $1997^{6}$. Esta mutación corresponde a un cambio de guanina a adenina en el nucleótido 1705 del ADN genómico del rLDL que se localiza en el intrón 11 y altera la secuencia de consenso GT altamente conservada que es necesaria para el correcto splicing del ARN mensajero, determinando la síntesis de un rLDL anormal. Específicamente, esta mutación afecta la región aminoacídica que circunda al residuo 545 de la secuencia proteica del rLDL, que corresponde al dominio extracelular que tiene homología con el precursor del receptor del factor de crecimiento epidérmi$\mathrm{co}^{2}$. Esta región del rLDL es importante tanto para la conformación correcta del dominio que permite la unión de las partículas de LDL, como para la posterior disociación de las $\mathrm{LDL}$ del receptor en los lisosomas que permite que el rLDL recicle hasta la superficie celular ${ }^{7}$. Por lo tanto, la mutación detectada en esta paciente produciría un rLDL con deficiencia funcional parcial, aunque no absoluta.

En forma interesante, esta mutación permite comprender la excelente respuesta de esta paciente al uso de terapia hipolipemiante con simvastatina en dosis alta combinada con ezetimiba, en comparación con otras variantes genéticas de HF mucho más resistentes al tratamiento farmacológi$\mathrm{Co}^{8}$. Dado que este caso clínico correspondería a una paciente con un rLDL con funcionalidad disminuida, pero con actividad funcional residual, esta deficiencia puede ser compensada por una mayor expresión del gen del rLDL por activación transcripcional secundaria a la depleción celular de colesterol determinada por el uso combinado de estatinas y ezetimiba. Por lo tanto, este caso es consistente con la variabilidad clínica que se observa en la respuesta de los pacientes con HF a las estatinas dependiendo de la heterogeneidad de las mutaciones presentes en el gen del $r L D L^{8}$.

Clásicamente, y como lo demuestra la evolución de esta paciente, la HF es una hiperlipemia de difícil manejo ${ }^{9}$. En este caso, la adición de ezetimiba al tratamiento con simvastatina, ha conseguido una reducción adicional en el c-LDL de $20 \%$, logrando por primera vez que la paciente alcance una concentración de c-LDL $<200 \mathrm{mg} / \mathrm{dl}$ de forma estable (Figura 1B). Esta terapia asociada es muy efectiva porque permite bloquear tanto la síntesis endógena como la absorción intestinal de colesterol $^{10}$. De hecho, la eficacia de la combinación de simvastatina/ezetimiba en el manejo de la HF heterocigota ya ha sido descrita ${ }^{11}$. Por lo tanto, esta aproximación farmacológica, la cual podría incluir el uso de otras estatinas más potentes como atorvastastina o rosuvastatina, puede postularse como alternativa terapéutica de primera elección para el manejo de estos pacientes. El estudio ENHANCE en curso deberá establecer el impacto de la terapia combinada en la evolución de la ateroesclerosis en pacientes con HF heterocigota $^{12}$.

También se ha evaluado la utilidad del tratamiento de estatina + ezetimiba en HF homocigota. Así, esta terapia produce un beneficio adicional a la monoterapia con estatinas en dosis altas en estos pacientes ${ }^{13}$. Por otro lado, la asociación de ezetimiba con dosis altas de estatinas ha demostrado ser más eficaz en controlar la hipercolesterolemia de rebote que se produce en los pacientes con HF homocigota postratamiento con LDL afére$\operatorname{sis}^{14}$.

La morbimortalidad cardiovascular en los pacientes con hipercolesterolemia familiar severa es muy alta $^{2}$. Este caso nos muestra como los pacientes con HF se pueden beneficiar utilizando la máxima capacidad terapéutica hipolipemiante disponible en la actualidad, lo que disminuiría significativamente el riesgo cardiovascular. Además, algunos pacientes portadores de HF, como lo sugiere este caso, parecen presentar mecanismos protectores (dependientes o independientes del metabolismo lipídico) frente al desarrollo de enfermedad aterosclerótica clínica ${ }^{15}$ que ameritan estudio adicional.

Finalmente, queremos insistir que la identificación temprana asociada a un tratamiento hipolipe- 
miante eficaz se traduce en un pronóstico vital significativamente mejor en los pacientes con $\mathrm{HF}^{2}$. Así mismo, la disponibilidad actual de métodos diagnósticos para la identificación del defecto

\section{REFERENCIAS}

1. Hobbs HH, Brown MS, Goldstein JL Molecular genetics of the LDL receptor gene in familial hypercholesterolemia. Hum Mutat 1992; 1: 44566.

2. Goldstein JL, Hobbs HH, Brown MS. Familial Hypercholesterolemia. In: Scriver CR, Beaudet AL, Sly WS, Valle D, eds. The Metabolic and Molecular Bases of Inherited Disease. $8^{\text {th }}$ ed. New York, NY: McGraw-Hill; 2001; 2863-2913.

3. Austin MA, Hutter CM, ZimMERn RL, Humphries SE. Genetic causes of monogenic heterozygous familial hypercholesterolemia: a HuGE prevalence review. Am J Epidemiol 2004; 160: 407-20.

4. Nervi F, Arteaga A, Maiz A, Tocornal J, Severin MC. Hipercolesterolemia familiar homocigota. Rev Méd Chile 1979; 107: 624-8.

5. Tejedor D, CastiLo S, Mozas P, JimÉnez E, López M, Tejedor MT et aL. Spanish FH Group. Reliable low-density DNA array based on allele-specific probes for detection of 118 mutations causing familial hypercholesterolemia. Clin Chem 2005; 51: 1137-44.

6. The low density lipoprotein receptor database. http://www.ucl.ac.uk/fh

7. Jeon H, Blacklow SC. Structure and physiologic function of the low-density lipoprotein receptor. Annu Rev Biochem 2005; 74: 535-462 ??

8. Miltiadus G, Xenophontos S, Bairaktari E, Ganotakis M, Cariolou M, Elisaf M. Genetic and environmental factors affecting the response to statin therapy in patients with molecularly defined familial hypercholesterolaemia. Pharmacogenet Genomics 2005; 15: 219-25. molecular en los pacientes con HF permitirá definir un tratamiento más preciso y efectivo de esta condición de muy alto riesgo cardiovascular.

9. Naoumova RP, Thompson GR, Soutar AK. Current management of severe homozygous hypercholesterolaemias. Curr Opin Lipidol 2004; 15: 413-22.

10. MuRDoch D, Scotт LJ. Ezetimibe/Simvastatin: a review of its use in the management of hypercholesterolemia. Am J Cardiovasc Drugs 2004; 4: 405-22.

11. Stein E, Stender S, Mata P, Sager P, Ponsonnet D, MeIani L et al. Ezetimibe Study Group. Achieving lipoprotein goals in patients at high risk with severe hypercholesterolemia: efficacy and safety of ezetimibe co-administered with atorvastatin. Am Heart J 2004; 148: 447-55.

12. Kastelein JJ, Sager PT, De Groot E, Veltri E. Comparison of ezetimibe plus simvastatin versus simvastatin monotherapy on atherosclemosis progression in familial hypercholesterolemia. Design and rationale of the Ezetimibe and Simvastatin in Hypercholesterolemia Enhances Atherosclerosis Regression (ENHANCE) trial. Am Heart J 2005; 149: 234-9.

13. Gagne C, Gaudet D, Bruckert E. Ezetimibe Study Group. Efficacy and safety of ezetimibe coadministered with atorvastatin or simvastatin in patients with homozygous familial hypercholesterolemia. Circulation 2002; 105: 2469-75.

14. Yamamoto A, Harada-Shiba M, Endo M, Kusakabe $\mathrm{N}$, TaNioka T, Kato H et al. The effect of ezetimibe on serum lipids and lipoproteins in patients with homozygous familial hypercholesterolemia undergoing LDL-apheresis therapy. Atherosclerosis 2006; 186: 126-31.

15. Hobbs HH, Graf GA, Yu L, Wilund KR, Cohen JC. Genetic defenses against hypercholesterolemia. Cold Spring Harb Symp Quant Biol 2002; 67: 499505.

\section{Agradecimientos}

El estudio genético de esta paciente ha sido posible gracias a la colaboración entre la Universidad de Valparaíso y la Fundación Española de Hipercolesterolemia Familiar. 